\title{
Technical Access in Blood Glucose Detection using ANN
}

\author{
F.Emerson Solomon, R.Kishore Kanna, Vasukidevi Ramachandran, S.Geetha
}

\begin{abstract}
Early re-affirmation of patients builds the expense of human services and it exceptionally impacts the notoriety of the clinic. Discovering readmission in essential stage, enables the clinics to give extraordinary consideration for those patients, and after that can lessen the rate of readmission. In this work build up another model utilizing profound learning. It is the correlation technique between AI and profound learning. Typically, Logistic relapse is utilized for all sort of expectation. Be that as it may, as per this information fake neural system model in profound learning give promising outcome than strategic relapse.
\end{abstract}

Keywords:Artificial neural network, Multilayer perception, Logistic Regression

\section{INTRODUCTION}

In the United States, practically 30.2 million individuals enduring diabetes perish. The figure speaks to among 27.9 and 32.7percent of the all out populace. Diabetes is the huge issue in social insurance world. It is way of life expire. Diabetes is considered as the perilous and unending infections because of increment in sugar level in blood. Heaps of inconveniences may happen if diabetes stays unidentified and untreated. The major distinguishing procedure is visiting a patient to a demonstrative focus and interview with a specialist. Anticipate diabetes by altering ways of life and intercession of new medications has convincingly demonstrated that these measures can.

Diabetic patient's emergency clinic readmission is a significant issue in human services industry. BeataStrack et.al (2014) learned about this point and they grew new model for foreseeing emergency clinic readmission utilizing strategic relapse. They predominantly centre around Hba1c result for their examination ${ }^{1}$.

A few productions are accessible identified with AI and neural system Sushant Ramesh et.al (2017) says diabetic expectation utilizing profound learning, with assistance of prima Indian diabetic informational index. Usage utilizing tensor stream edge work. Counts done by the assistance of Random Forest Algorithm. Alongside they are utilizing profound neural system model for foreseeing the diabetic patients [3]. Different distributed papers learned about patient's emergency clinic confirmations. Ahmad Hammoudeh et.al (2018) utilizes CNN model for order purposes. The casing work they are utilized are Tensor

Revised Manuscript Received on July 18, 2019.

DR.F.Emerson Solomon, Dept Of Biomedical Engg, BIHER, Chennai,Tamilnadu, India.

R.Kishore Kanna, Dept Of Biomedical Engg, BIHER, ChennaiTamilnadu, India.

DR.Vasukidevi Ramachandran, Dept Of Biomedical Engg, BIHER,Chennai, Tamilnadu, India.

S.Geetha, Dept Of Biomedical Engg, BIHER, Chennai, Tamilnadu, India. stream and scikit learn for neural system usage. Mix of convolutional neuralsystem model and information building give promising outcome for restorative fields .

Bhuvan M S et.al (2016) learned about the medicinal information examination through partner guideline mining and highlight significance mining alongside they learned about characterization calculations identified with these informational indexes. In light of the examination finding the hazard variables impacts the readmission dependent on the diabetic information ${ }^{2}$.

\section{WORKING METHODOLOGY}

Working Data

The dataset taken from UCI Machine Learning Repository, it is a major dataset with over more than one need one hundred lines and 55 segment that is properties, removed from patient's wellbeing records from United States more than 150 emergency clinics from 1999-2008, each line considered as an experience record of every patient. Experience Record incorporates encounter, admission_type_id_description, discharge_disposition_id portrayal, confirmation sourcedescriptions,time_in_hospital , num_lab_procedures, and analysis result in 3 phases, aftereffect of A1C Test and furthermore 23 diabetic medications. 23 medications incorporate basic diabetic prescriptions like metformin, glimepiride etc. those are fundamental prescription. If there should be an occurrence of genuine diabetic cases specialists may give blends of prescriptions most significant piece of this informational index is a few highlights contains subdivisions. If there should be an occurrence of Discharge portrayal id and affirmation source id depictions and so on.

Restorative records of every patient included in excess of 50 hazard components and a one segment contains class name speaks to whether a patient was re-conceded or not. Name contains 3 classes readmission inside multi day time span, following thirty days or patient was never readmitted. From the information the readmission rate is as per the following 35 level of patients were readmitted inside one month54 rate patients were not readmitted ${ }^{4}$.

Analysis is the technique for a patient is diabetic or not. $\mathrm{HbA} 1 \mathrm{c}$ test is the best technique for diabetic discovery. It is Haemoglobin A1c test it clarifies the normal glucose dimension of a patient over past a few months. It is otherwise called HbA1c Glycated Haemoglobin test. Typical dimension of $\mathrm{HbA} 1 \mathrm{c}$ test is $4 \%$ to $5.6 \%$ and $5.7 \%$ to 
$6.4 \%$ says that those individuals have high shot of getting a diabetic patient. $6.5 \%$ or more shows they have diabetics. The higher A1c result demonstrates the hazard having muddled identified with diabetic patients ${ }^{5}$

Table 1 shows missing qualities in the information. Information contains one need one thousand and fifty-six experience. claim to fame and payer code contains high level of missing qualities. Expelling these traits from the information in the pre-processing stage. Payer code is the one of the components in the information it's anything but a significant ${ }^{6}$.

Table 1. List containing missing values

\begin{tabular}{|l|l|}
\hline Attribute & Missing values \\
\hline Race & $2.23 \%$ \\
\hline Weight & $96.85 \%$ \\
\hline Payer Code & $40.5 \%$ \\
\hline Medical Specialty & $50.1 \%$ \\
\hline Diag_1 & $.02 \%$ \\
\hline Diag_2 & $.35 \%$ \\
\hline Diag_3 & $1 \%$ \\
\hline
\end{tabular}

Sexual orientation having less no of missing qualities. Information contains three finding result. On the off chance that multiple highlights are absent in one experience it is considered as awful information, so evacuate an experience whether 3 analysis are vacant.

Table 2: ICD9 coded and difficulties

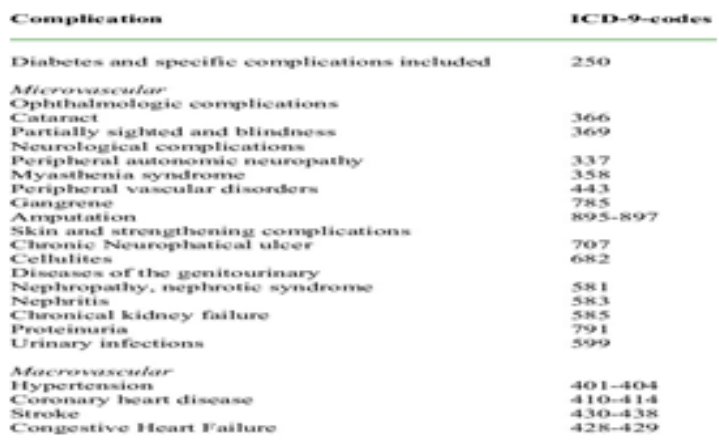

ICD9 codes resemble medicinal coding. Utilized for speaking to illness in restorative industry. Codes incorporate qualities from 001 to 999 . Each code speaks to every sickness. 250.00 to 250.99 speaks to icd9 code of diabetic mellitus. Table 2 speaks to icd 9 code and comparing sickness.

\section{STRUCTURE OF SYSTEM}

Design of proposed model appeared in fig 1. Information contains bunches of missing qualities, insignificant information and clamours so pre-processing is the significant advance. In pre-processing step information standardize utilizing likelihood dissemination. Information split in to test set and train set. Here $80 \%$ information utilized for preparing and $20 \%$ information utilized for testing. Model structure is utilizing mlp neural

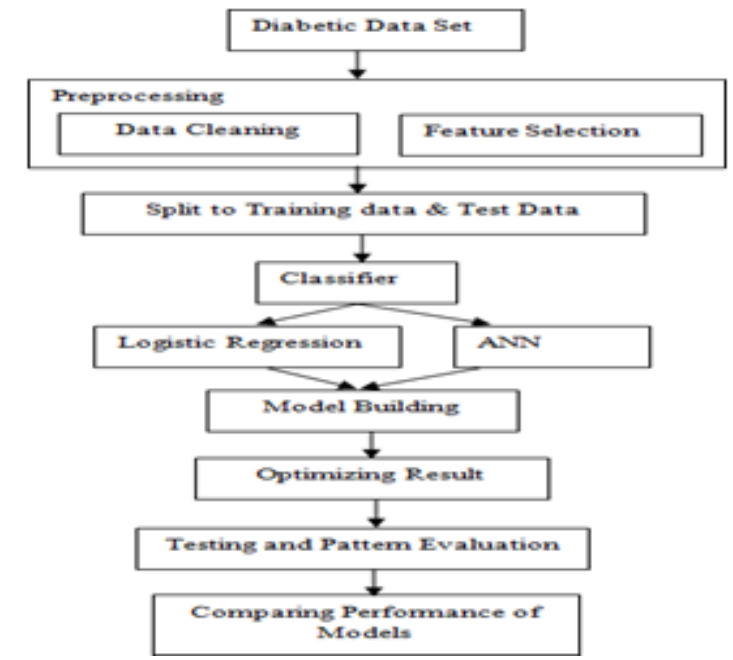

Fig 1: Structure Of System

system and finally looking at the model exactness utilizing existing and proposed strategy. For information approval tenfold cross approval strategies are utilized.

\section{IV.LOGISTIC DATA REGRESSION}

Calculated relapse is famously known as AI grouping technique for forecast. Strategic relapse contains a reliant variable it is otherwise called target variable.

Autonomous variable is otherwise called Predictor variable. Relapse investigation is a significant apparatus for dissecting and displaying information.

Condition 1 demonstrates the fundamental condition of the straight relapse. Every other condition are driven from this essential condition. It is prevalently known as the condition of the straight line. Adding sigmoid capacity to the straight relapse we can grow new condition for the calculated relapse.

Counterfeit neural systems (ANN) is figuring frameworks. It created by getting the thought from the structure of natural neural systems that that considered as human brain. Neuralsystem organize itself is never considered as an ideal calculation;however it is a structure helps a wide range of Machine

Learning calculations to process together to run and unravel complex information inputs. ANN contains counterfeit neurons. Fake neurons mean set of associated hubs or units, which impersonates the neurons in a human mind. Every single association same as the neurotransmitters in mind, can transmits signals starting with one neuron then onto the next neuron. At the point when counterfeit neuron gets a sign it will process the sign and act as needs be. 
International Journal of Innovative Technology and Exploring Engineering (IJITEE) ISSN: 2278-3075, Volume-8, Issue- 9S2, July 2019

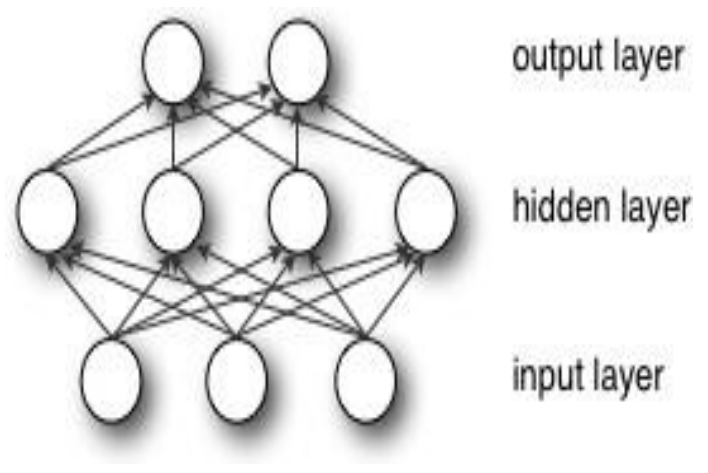

Fig. 2 Layers of neural network

Multilayer perceptron (MLP) is a solitary shrouded layer. It is significant and established sort of counterfeit neural system model. MLP contains 3 layers of hubs: first info layer, shrouded layer and the last layer is yield layer. In this every hub is considered as neuron that uses a nonlinear actuation work. On the off chance that layers in MLP are associated, every hub in each layer is associated with explicit weight. It uses back spread calculation. MLP calculations for the most part used to take care of the arrangement forecast issue and furthermore characterization relapse issue.

\section{BODY OF MODEL}

AI can manage colossal measure of information. However, Deep Learning is exceptionally productive for arrangements with that information. Fabricate another model utilizing profound learning. Profound learning contains bunches of calculation for grouping here utilizing Multilayer perceptron Artificial neural system model for growing new model $^{3}$.

\section{RESULTS}

Calculated Regression Result

\begin{tabular}{|l|l|}
\hline $\begin{array}{l}\text { Original dataset shape } \\
\text { Counter }\end{array}$ & $(\{0: 56476,1: 5199\})$ \\
\hline $\begin{array}{l}\text { New dataset shape } \\
\text { Counter }\end{array}$ & $(\{0: 56476,1: 56476\})$ \\
\hline No. Observations & 90361 \\
\hline $\begin{array}{l}\text { Cross Validation } \\
\text { Score: }\end{array}$ & $60.70 \%$ \\
\hline Dev Set score: & $60.59 \%$ \\
\hline Accuracy & $61.62 \%$ \\
\hline $\begin{array}{l}\text { Current function } \\
\text { value: }\end{array}$ & 0.665612 \\
\hline Iterations: & 35 \\
\hline
\end{tabular}

Table 3: Result of MLP Artificial neural network Model

\begin{tabular}{|l|l|}
\hline $\begin{array}{l}\text { Original dataset shape } \\
\text { Counter }\end{array}$ & $(\{0: 56476,1: 5199\})$ \\
\hline $\begin{array}{l}\text { New dataset shape } \\
\text { Counter }\end{array}$ & $(\{0: 56476,1: 56476\})$ \\
\hline No. Observations & 90361 \\
\hline Dev Set score: & $81.63 \%$ \\
\hline Accuracy & $82.86 \%$ \\
\hline F1 score & $82 \%$ \\
\hline
\end{tabular}

Table 4 :Result Comparison between Logistic Regression and MLP Neural network.

Contains Dataset subtleties Accuracy and cross approval score from the investigation Table contains informational index shape, Accuracy,

Deviation score and $\mathrm{f} 1$ score of disarray network.

\begin{tabular}{l|l|l|l|}
\hline Model & Accuracy & Precision & Recall \\
\hline $\begin{array}{l}\text { Logistic } \\
\text { Regression }\end{array}$ & 61.62 & 62 & 55 \\
\hline $\begin{array}{l}\text { MLPNeural } \\
\text { Network }\end{array}$ & $\mathbf{8 2 . 8 6}$ & $\mathbf{8 2}$ & $\mathbf{8 0}$ \\
\hline
\end{tabular}

Table 4 shows the comparison between two methods. It compares Accuracy and Precision recall scores.

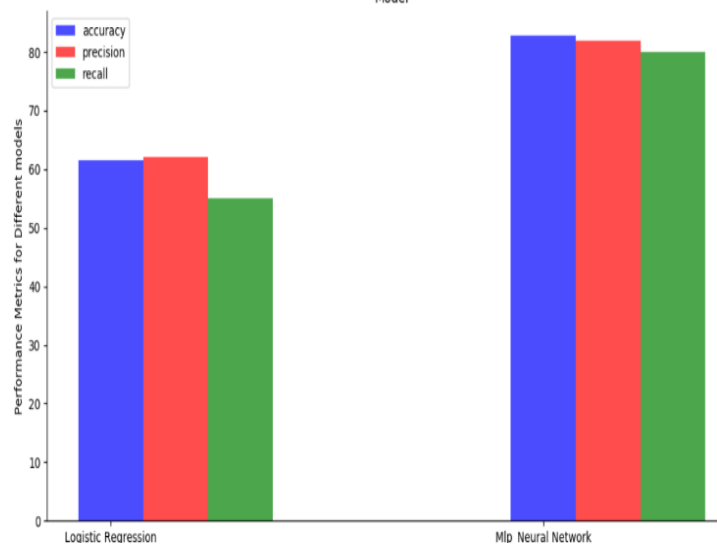

Fig 3: Graphical representation of performance matrix

\section{CONCLUSION}

Proposed model gives promising outcome than the current model. Here we are anticipating early readmission of diabetic patients utilizing ai and profound learning arrangement algorithms. Multilayer perceptron artificial neural system model gives better precision and lower blunder rate than customary foreseeing technique strategic relapse 


\section{REFERENCES}

1. Ramesh, S., Caytiles, R. D., \&Iyengar, N. C. S. (2017). A Deep Learning Approach to Identify Diabetes. ADV SCI Technology Letter, 145,44-49

2. Safavian, S. R., \&Landgrebe, D. (1991). A survey of decision tree classifier methodology. IEEE transactions on systems, man, and cybernetics, 21(3),660-674.

3. Chan, J. C. W., \&Paelinckx, D. (2008). Evaluation of Random Forest and Adaboost tree-based ensemble classification and spectral band selection for ecotopemapping using airborne hyperspectral imagery. REMOTE SENS ENVIRON, 112(6),2999-3011.

4. Pearce, J., \& Ferrier, S. (2000). Evaluating the predictive performance of habitat models developed using logistic regression. ECOL MODEL, 133(3),225-245.

5. Mogensen, C. E., \& Christensen, C. K. (1984). Predicting diabetic nephropathy in insulin-dependent patients. NEW ENGL J MED, 311(2),89-93.

6. Kansagara, D., Englander, H., Salanitro, A., Kagen, D., Theobald, C., Freeman, M., \&Kripalani, S. (2011). Risk prediction models for hospital readmission: a systematic review. Jama, 306(15),1688-1698. 